compared to subjects without, have lower lung volumes (upright and supine), a greater airway impedance (seen in FOT at FRC, both upright and supine), and a larger fall in their ERV on lying down. All these differences may be due to the higher BMI, and in particular differences in distribution of fat in subjects with early chronic respiratory failure. However, these obesity differences were not reflected in large differences in the AHI or ODI between the groups.

\section{S118 THE VENTILATORY RESPONSE TO CO2 WITHIN OBSTRUCTIVE SLEEP APNEA PATIENTS}

${ }^{1} \mathrm{CMN}$ Earing, ${ }^{1} \mathrm{JP}$ Moore, ${ }^{2} \mathrm{DJ}$ McKeon, ${ }^{1} \mathrm{H}$-P Kubis; ${ }^{1}$ Bangor University, Bangor, Gwynedd; ${ }^{2}$ Respiratory Department, Ysbyty Gwynedd, Bangor, Gwynedd

\subsection{6/thoraxjnl-2013-204457.125}

Introduction Obstructive Sleep Apnea (OSA) is a condition defined by the collapse of the upper airway and cessation of respiration during sleep. The resulting hypoventilation leads to intermittent nocturnal hypoxia and increased arterial $\mathrm{pCO}_{2}$ (hypercapnia). Control of ventilation is largely dependent on two interactive pathways, the central and peripheral chemoreceptors. Both chemoreceptors' respond to $\mathrm{H}^{+}$ions liberated as a result of the presence of $\mathrm{CO}_{2}$. However, the peripheral chemoreceptors also respond to hypoxia particularly when $\mathrm{PaO}_{2}$ falls below 70 Torr. Hypoxia has a synergistic effect on the ventilatory response to $\mathrm{CO}_{2}$. Our study therefore investigated the ventilatory response to $\mathrm{CO}_{2}$ with and without the presence of moderate hypoxia to determine whether the central and/or peripheral chemoreflex response to $\mathrm{CO}_{2}$ is modified with the development of OSA.

Methods The ventilatory response to $\mathrm{CO}_{2}$ amongst 33 newly diagnosed male OSA patients (AHI: $32.6 \pm 24.8$; age: $53.1 \pm 10.3$ years, BMI: $\left.36.4 \pm 6.8 \mathrm{~kg} / \mathrm{m}^{2}\right)$ was measured whilst breathing four different gas mixtures balanced with $\mathrm{N}_{2}$ (Mixture 1: ambient air; mixture 2: $25 \% \mathrm{O}_{2} / 6 \% \mathrm{CO}_{2}$; mixture 3: $13 \%$ $\mathrm{O}_{2}$; mixture 4: $\left.13 \% \mathrm{O}_{2} / 6 \% \mathrm{CO}_{2}\right)$. The minute ventilation of each participant, normalised by their body surface area (BSA), was recorded with subjects blinded to the order of test gases. Additionally, fasted venous blood samples were taken to assess plasma leptin concentrations.

Results Mild and moderate OSA patients (AHI: $13.76 \pm 7.01$ ) revealed a significantly $(p<.01)$ greater change in their ventilatory response to the hyperoxic-hypercapnic gas mixture $\left(5.22 \pm 1.951 \mathrm{x} \mathrm{min}^{-1} \times \mathrm{BSA}^{-1}\right)$ compared to severe OSA patients (AHI: $55.31 \pm 18.25)\left(3.26 \pm 1.641 \times \min ^{-1} \times \mathrm{BSA}^{-1}\right)$. There was no significant change in ventilation between the hyperoxic and hypoxic-hypercapnic conditions in both groups. A significant negative correlation $(r=-0.39 ; p<.05)$ was found between AHI and ventilation change $\left(1 \times \min ^{-1} \times \mathrm{BSA}^{-1}\right)$ to the hyperoxic-hypercapnic gas mixture.

Conclusion These findings suggest the reduced ventilatory response to hypercapnia amongst severe OSA patients is likely a result of adaptation to the central chemoreceptors.

\section{S119 IS THE HYPERCAPNIC VENTILATORY RESPONSE STILL RELEVANT TO CENTRAL SLEEP APNOEA IN THE ERA OF MODERN HEART FAILURE MANAGEMENT?}

${ }^{1}$ A Atalla, ${ }^{1}$ TW Carlisle, ${ }^{2}$ AK Simonds, ${ }^{1}$ MR Cowie, ${ }^{1} \mathrm{MJ}$ Morrell ; ${ }^{1}$ National Heart and Lung Institute, Imperial College, London, United Kingdom; ${ }^{2}$ Academic Unit of Sleep and Breathing, Royal Brompton Hospital, London, United Kingdom

10.1136/thoraxjn-2013-204457.126
Introduction A brisk ventilatory response to carbon dioxide $\left(\mathrm{CO}_{2}\right)$ is integral to the development of central sleep disordered breathing (SDB) in heart failure (HF) patients. Modern treatments for HF enhance cardiac function and many improve central SDB. The role of hypercapnic ventilatory responses (HCVR) in central SDB in HF patients managed according to modern guidelines is unclear. For example, adaptive servoventilation (ASV), used to treat central SDB, both improves cardiac function and reduces hypercapnic ventilatory responses (HCVR), suggesting that heightened chemosensitivity in HF may relate to cardiac impairment.

Objective To test the hypothesis that there would be no difference in HCVR between optimally treated patients with HF and central SDB (HF-CSA), and those with HF alone (HF-noSDB). Method Measurements of resting expired $\mathrm{CO}_{2}$ (awake) and evening and morning HCVR, using the Read rebreathe technique, were undertaken in patients optimally treated for HF. Patients also underwent overnight polysomnography. Sample size calculations (using data from Javaheri, NEJM 1999) showed 10 patients were needed in each group. Statistical analyses were undertaken using SPSS. The study received ethical approval.

Results Twenty-six HF patients were studied (11 with HF-CSA: median (IQR) age 68 (58-78) and 15 with HF-noSDB: age 72 (67-78)years). Left ventricular ejection fraction was: HF-CSA 32 $(20-40) \%$ and HF-noSDB $40(27-47) \%$. The apnoea hypopnoea index was: HF-CSA $14.6(12.9-37.1) / \mathrm{hr}$ and HF-noSDB 5.0 (3.2-6.0)/hr. The HF-CSA group had lower median resting expired $\mathrm{CO}_{2}$ than the HF-noSDB group (end tidal $\mathrm{CO}_{2}: 30.6$ $(28.6-37.3)$ vs. $36.2(35.2-40.4) \mathrm{mmHg}, \mathrm{p}=0.02)$. There was no significant difference between the HF-CSA and HF-noSDB in evening HCVR (2.15 (1.70-2.74) vs. $1.99(1.60-3.33) \mathrm{L} / \mathrm{min} /$ mmHg ETCO $2, \mathrm{p}=0.53$ ) or morning HCVR (2.71 (1.43-4.88) vs. $\left.2.20(1.00-3.00) \mathrm{L} / \mathrm{min} / \mathrm{mmHg} \mathrm{ETCO}_{2}, \mathrm{p}=0.23\right)$. Resting expired $\mathrm{CO}_{2}$ in the total study population correlated negatively with morning, but not evening, HCVR.

Conclusion The results of this small study suggest that modern HF management may have an effect on ventilatory stability via changes in HCVR. The timing of the HCVR tests may be a factor. We speculate that overnight disturbances in breathing may promote ventilatory instability in the morning, rather than the evening.

\section{S120 HYPERPOLARISED 3HE DIFFUSION MRI AND MULTIPLE BREATH INERT GAS WASHOUT IN PATIENTS WITH ASTHMA}

${ }^{1} \mathrm{~S}$ Gonem, ${ }^{2} \mathrm{~S}$ Hardy, ${ }^{2} \mathrm{~N}$ Buhl, ${ }^{1} \mathrm{M}$ Soares, ${ }^{3} \mathrm{R}$ Costanza, ${ }^{4} \mathrm{P}$ Gustafsson, ${ }^{1} \mathrm{CE}$ Brightling, 2J Owers-Bradley, 'S Siddiqui; 'Institute for Lung Health, University of Leicester, Leicester, UK; ${ }^{2}$ Department of Physics and Astronomy, University of Nottingham, Nottingham, UK; ${ }^{3}$ Chiesi UK Ltd., Cheadle, UK; ${ }^{4}$ Department of Paediatrics, Central Hospital, Skövde, Sweden

\subsection{6/thoraxjnl-2013-204457.127}

Background Multiple breath inert gas washout (MBW) is a technique for detecting abnormal ventilation distribution in patients with asthma and other airway diseases. $S_{\text {cond }}$ and $S_{\text {acin }}$ are measures of convective-dependent inhomogeneity (CDI) and diffusion-convection-dependent inhomogeneity (DCDI) respectively. Hyperpolarised ${ }^{3} \mathrm{He}$ diffusion magnetic resonance imaging $\left({ }^{3} \mathrm{He}-\right.$ MRI) may be used to probe lung microstructure at a variety of length scales, with short timescale $(14 \mathrm{~ms})$ apparent diffusion coefficient (ADC) corresponding to diffusion within an alveolus or a single acinar airway, and long timescale $(1.5 \mathrm{~s}-6 \mathrm{~s})$ ADC 